Article

\title{
Entropy Change Caused by Martensitic Transformations of Ferromagnetic Shape Memory Alloys
}

\author{
Victor A. L'vov ${ }^{1,2}$ (D) , Eduard Cesari ${ }^{3}$ (D) ${ }^{\text {Anna Kosogor }}{ }^{2, *}$, Joan Torrens-Serra ${ }^{3}$, \\ Vicente Recarte ${ }^{4}$ (D) and José Ignacio Pérez-Landazábal ${ }^{4}$ \\ 1 Faculty of Radiophysics, Electronics and Computer Systems, Taras Shevchenko University, \\ Glushkov Str. 4G, Kyiv 01601, Ukraine; victor.a.lvov@gmail.com \\ 2 Department of Theory of Magnetic Phenomena and Magnetic Dynamics of Solid State, \\ Institute of Magnetism, 36-b, Vernadsky Str., Kyiv 03142, Ukraine \\ 3 Departament de Fisica, Universitat de les Illes Balears, Ctra. de Valldemossa, km 7.5, \\ Palma de Mallorca 07122, Spain; eduard.cesari@uib.cat (E.C.); j.torrens@uib.es (J.T.-S.) \\ 4 Departamento de Física, Universidad Pública de Navarra (UPNA), Campus Arrosadia, Pamplona 31006, \\ Spain; recarte@unavarra.es (V.R.); ipzlanda@unavarra.es (J.I.P.-L.) \\ * Correspondence: annakosogor@gmail.com; Tel.: +380-98-555-47-57
}

Received: 26 October 2017; Accepted: 13 November 2017; Published: 19 November 2017

\begin{abstract}
In this paper, our most recent findings on the influence of magnetic order on the main transformational caloric and elastic properties of shape memory alloys (SMAs) are reviewed. It is argued that ferromagnetic order has a strong influence on the temperature interval of martensitic transformation (MT), the characteristics of stress-induced MT, and the shear elastic modulus of SMA. The problem of separation of the magnetic contributions to the entropy change $\Delta S$ and heat $Q$ exchanged in the course of martensitic transformation (MT) of SMA is considered in general terms, and theoretical formulas enabling the solution of the problem are presented. As an example, the $\Delta S$ and $Q$ values, which were experimentally determined for Ni-Mn-Ga and Ni-Fe-Ga alloys with different Curie temperatures $T_{C}$ and MT temperatures $T_{M}$, are theoretically analyzed. It is shown that for Ni-Mn-Ga martensites with $T_{M}<T_{C}$, the ratio of elastic and magnetic contributions to the entropy change may be greater or smaller than unity, depending on the temperature difference $T_{C}-T_{M}$.
\end{abstract}

Keywords: shape memory alloys; transformational properties; magnetic order; entropy change; elastic modulus

\section{Introduction}

Magnetic shape memory alloys exhibit a thermoelastic martensitic transformation (MT) from the high-symmetry phase (austenite) to the low-symmetry state (martensite) and, related to this transformation, display several functional properties. For example, large strains are observed under application of a magnetic field to the martensite due to the reorientation of martensite variants in the so-called "conventional" ferromagnetic shape memory alloys (FSMAs), like Ni-Mn-Ga and Ni-Fe-Ga [1-3].

In alloys such as Ni-Mn- $X(X=\mathrm{In}, \mathrm{Sn}, \mathrm{Sb})$, the MT usually takes place between ferromagnetic austenite and weakly magnetic martensite; thus, the application of a magnetic field makes the reverse MT possible, which is referred to as the magnetic shape memory effect, or the metamagnetic behavior of the alloy [4]. Due to large differences in magnetization values between the phases involved in the MT, the metamagnetic alloys show interesting and promising properties, such as high values of magnetoresistance [5] and inverse magnetocaloric effect [6]. In connection with this, the entropy change, $\Delta S$, accompanying the MT for different metamagnetic alloys has been measured (see [7] and 
references therein). In order to clarify the notation, we note that $S_{M}<S_{A}$ and consider the positive values $\Delta S=\left|S_{M}-S_{A}\right|$, where subscripts $A$ and $M$ stand for austenite and martensite, respectively. Then, the exchanged heat is $Q \leq T_{M} \Delta S$, where $T_{M}$ is MT temperature. (The equality $Q=T_{M} \Delta S$ is fulfilled in the ideal case where the MT follows a reversible path between equilibrium phases.) In some metamagnetic alloys, particularly Ni-Mn-In and Ni-Mn-In-Co, a strong dependence of the $\Delta S$ value on the composition and/or the degree of chemical order of the alloy has been observed [8,9]. Moreover, the entropy change $\Delta S$, measured for alloys with different values of Curie temperature of austenite, $T_{C A}$, and MT temperature, strongly increases with the decrease of interval between $T_{C A}$ and $T_{M}$ (for the purposes discussed here, $T_{M}$ can be defined as the peak temperature in a typical differential scanning calorimetry (DSC) run). Considering the $\Delta S$ value formed by an elastic/lattice contribution, $\Delta S_{e l}$, and a magnetic contribution, $\Delta S_{\text {mag }}$, it was found that the increase of $\Delta S$ can be attributed to the decrease of $\Delta S_{\text {mag }}\left(\Delta S_{\text {mag }}>0 ; \Delta S=\left|\Delta S_{\text {el }}\right|-\left|\Delta S_{\text {mag }}\right|\right)$ [9]. Very recently, it has been clarified that this evolution is not directly related to a chemical (atomic) ordering effect, but to the higher magnetic order of austenite as $T_{M}$ goes below $T_{C A}$ [10]. In FSMAs such as Ni-Mn-Ga and Ni-Fe-Ga, the MT can take place between ferromagnetic phases, if $T_{M}<T_{C A}$ or, for compositions with $T_{M}>T_{C A}$ and $T_{M}>T_{C M}$, both phases are paramagnetic. In contrast to metamagnetic transformations, the MTs in these SMAs are not accompanied by a drastic change in the magnetic ordering; therefore, the magnetization change during MT is small, and the contributions of their magnetic subsystems to the entropy change, $\Delta S_{\text {mag }}$, have to be different from those observed for metamagnetic alloys.

In this paper, we review our most recent findings on the influence of magnetic order on the main transformational properties of FSMAs, citing as an example the Ni-Mn-Ga alloy system, and we discuss both the measured and theoretically evaluated magnetic contributions to the entropy change $\Delta S$ and heat exchange $Q$ in the course of MT of FSMA for different families of Ni-Mn-Ga and Ni-Fe-Ga alloys. The analysis of experimental data is based on the Landau expansion for the Helmholtz free energy of FSMA, which has formerly been used for the description of the thermodynamic properties of different shape memory alloys; therefore, the main principles of this analysis are applicable to other FSMAs, as well.

\section{Influence of Ferromagnetic Order on the Transformational Properties of FSMAs}

Due to magnetoelastic coupling, the ferromagnetic order influences the MT strains, elastic moduli, thermal properties of shape memory alloys, etc. The interrelation between the ferromagnetic order and transformational properties of FSMAs has been studied for the case of MT from the cubic phase to tetragonal phase characterized by the lattice parameters $a=b$ and $c$ (see the review article [11] and references therein). To describe this interrelation quantitatively, the Landau expansion for the Gibbs potential has been expressed through the order parameter of MT, $u=2(c / a-1)$, and the relative volume change of deformed solid, $v$. The expansion includes the magnetoelastic term $\delta_{\text {ex }} v M^{2}(T) / 3$, which describes, in particular, a spontaneous volume magnetostriction that arises below Curie temperature $T_{C A}$ due to ferromagnetic ordering $\left(\delta_{e x}\right.$ is the magnetoelastic constant, $M(T)$ is a temperature-dependent magnetization value). It appears that the difference between the coefficients $c_{2}^{f m}$ and $c_{2}$ of the quadratic terms of Landau expansion for the Gibbs potential of ferromagnetic and paramagnetic states, respectively, can be estimated as $c_{2}^{f m}-c_{2} \approx 10 \delta_{e x} M^{2}(T) \approx 4$ GPa for Ni-Mn-Ga (see [12] for details). Taking into account that $c_{2}^{f m}=C^{\prime} / 3$ and the shear elastic modulus $C^{\prime}$ is of the order of $10 \mathrm{GPa}$ if the temperature of alloy is close to $T_{M S}$, it can be concluded that the magnetic order has an important effect on the elastic moduli of FSMA. This effect was observed experimentally and described theoretically in Refs. [13,14]. It was shown that the abrupt changes of the temperature derivatives of elastic moduli observed for the Ni-Mn-Ga alloys with $T_{M}<T_{C A}$ in the vicinity of their Curie points are induced by spontaneous volume magnetostriction.

The dependence of the coefficients in the expression for Gibbs potential on magnetization of FSMA may explain the influence of the magnetic order on the transformational properties of these 
alloys, because the MT strain can be expressed through these coefficients. As such, the MT strains depend on magnetization value if the MT takes place in the ferromagnetic state [11].

It is worth noting that when the magnetoelastic coupling is taken into account, the renormalization of coefficients in the expression for Gibbs potential does not result in any change in its mathematical form. Therefore, the magnetic order does not produce the appearance of qualitatively new features in the MT, but quantitatively changes its characteristics. Accordingly, a quantitative theoretical analysis of existing experimental results and specially planned additional experiments are needed for the estimation of the magnetic and non-magnetic contributions to the physical characteristics of MT. This analysis was carried out for the Ni-Mn-Ga alloy system as explained in Section 3.

In order to establish and further clarify the role of magnetic contributions to the entropy change and heat exchange in the course of MT, the thermodynamic properties of Ni-Mn-Ga alloys exhibiting martensitic transformation above and below the Curie temperature of austenite have been compared [12]. Several experimental results show noticeable differences in the structural, thermomechanical, elastic and magnetic properties between low-temperature alloys (LTAs), with $T_{M}<T_{C A}, T_{M}<T_{C M}$, and high-temperature alloys (HTA), with $T_{M}>T_{C M}$. The LTAs undergo the MT from ferromagnetic cubic phase to ferromagnetic modulated tetragonal phase with $c / a<1$ on cooling, whereas MT takes place in the paramagnetic state of martensite in HTAs, and leads to formation of non-modulated tetragonal martensite with $c / a>1$. The absolute values of transformation heat are clearly lower for LTAs compared to those measured for HTAs [1,15-17]. This is an example of the interrelation between the ferromagnetic ordering and the transformational properties of Ni-Mn-Ga alloys. Another example of this kind was discovered long ago. It was observed that the MT in LTAs is accompanied by a jump-like change of magnetization [18]. This effect is related to (i) the relative volume change during $\mathrm{MT}, v_{M T}$, and (ii) the magnetoelastic coupling pressent in all magnetic solids [19], and is responsible for the emergence of the magnetic contributions of $\Delta S$ and $Q$ values as it will be described in the next section. Furthermore, the magnetic subsystem is able to make the main contribution to these values when the MT temperature is close to $T_{C A}$ [7]. In contrast to LTAs, the magnetic subsystem does not contribute so noticeably to $\Delta S$ and $Q$ in HTAs.

\section{Evaluation of Magnetic and Non-Magnetic Contributions to $\Delta S$ and $Q$}

We have argued above that the elastic properties of shape memory alloys in the ferromagnetic phase depend on magnetic order; therefore, we will consider that $\Delta S_{\text {mag }}$ is the part of $\Delta S$ that depends on spontaneous magnetization arising below $T_{C A}$. Therefore, the elastic contribution is $\Delta S_{e l}=\Delta S-\Delta S_{\text {mag }}$.

Let the forward MT start on cooling of alloy, when $T=T_{M S}$, and finish when $T=T_{M F}$. In Reference [7], the following expressions were obtained:

$$
\begin{gathered}
\Delta S_{e l}=\frac{1}{2} u^{2}\left(T_{M F}\right)\left(\frac{\partial c_{2}^{f m}}{d T}\right)_{T=T_{M F}}, \\
\Delta S_{m a g}=-\frac{2}{3} \delta_{e x} v_{M T} M\left(T_{M F}\right)\left(\frac{\partial M}{\partial T}\right)_{T=T_{M F}},
\end{gathered}
$$

with $v_{M T}=\left(V_{M}-V_{A}\right) / V_{A}$ representing the relative volume change, and $T_{M F}$ the martensite finish transformation temperature.

The derivative $d c_{2}^{f m} / d T$ can be evaluated through the temperature dependence of the critical stress $\sigma_{M S}$, which is needed to start a stress-induced MT, as

$$
\left(\frac{d c_{2}^{f m}}{d T}\right)_{T=T_{M S}}=\frac{1}{3 g u\left(T_{M F}\right)} \frac{d \sigma_{M S}}{d T}
$$


where the dimensionless coefficient $g$ depends on the sign of $1-c / a$ in martensite and on the direction/mode of axial loading [12]. Taking into account that $c_{2}^{f m}(T)$ depends linearly on temperature, and assuming that its slope is constant between the martensite starting and finishing transformation temperatures, $T_{M S}$ and $T_{M F}$, we can include the above expression for $d c_{2}^{f m} / d T$ in Equation (1), obtaining:

$$
\Delta S=\frac{1}{6 g} u\left(T_{M F}\right) \frac{d \sigma_{M S}}{d T}=\frac{1}{3 g}\left(\frac{c}{a}-1\right) \frac{d \sigma_{M S}}{d T} .
$$

It is noteworthy that in cases where both austenitic and martensitic phases are ferromagnetic, the entropy change estimated from Equation (4), as well as the experimental values of the derivative $d \sigma_{M S} / d T$, are the sum of elastic and magnetic parts, because Equation (3) relates this derivative to the coefficient $c_{2}^{f m}$ of the second-order term in the Helmholtz free energy of ferromagnetic phase, which includes a magnetic summand $\propto \delta_{e x} M^{2}$ (see above).

For the phase transition between equilibrium thermodynamic states, the transformation heat $Q$ is equal to the maximal possible value $Q_{\max }=T_{M} \Delta S$, where $T_{M}$ can be estimated as $T_{M}=\left(T_{M S}+T_{M F}\right) / 2$. Real martensitic state is not entirely equilibrium, i.e., its energy is higher then the energy of the equilibrium state, and so, $Q<Q_{\max }$. Therefore, it is instructive to compare the measured transformation heat $Q$, e.g., measured by DSC, which fulfills the inequality $Q / T_{M} \leq \Delta S$, with the calculated heat values $Q_{\text {calc }}=Q_{\max }$, obtained from $\Delta S_{\text {calc }}$ through its dependence on $d \sigma_{M S} / d T$.

Table 1 shows the collected values of $Q, Q_{\text {calc }}, \Delta S / m$ and $\Delta S_{\text {calc }} / m$ for different Ni-Mn-Ga alloys from Reference [12]. It is worth noting the generally good agreement between $\Delta S$ and $\Delta S_{\text {calc }}$, as well as the coherence between the values of $Q$ and $Q_{\text {calc }}$.

Table 1. DSC-measured transformation heat $Q$, calculated transformation heat $Q_{\text {calc, }}$, estimated entropy change by unit of mass $\Delta S / m$, and calculated entropy change by unit of mass $\Delta S_{\text {calc }} / m$ for different $\mathrm{Ni}-\mathrm{Mn}-\mathrm{Ga}$ alloys. The a) and b) cases denote the axis direction along which the compression force was applied, as explained in the text.

\begin{tabular}{|c|c|c|c|c|c|}
\hline No. & Alloy & $Q(\mathrm{~J} / \mathrm{g}) \mathrm{DSC}$ & $Q_{\text {calc }}(\mathrm{J} / \mathrm{g})$ & $\Delta S / m(\mathrm{~J} / \mathrm{kg} \cdot \mathrm{K})$ & $\Delta S_{\text {calc }} / m(\mathrm{~J} / \mathrm{kg} \cdot \mathrm{K})$ \\
\hline 1 & $\mathrm{Ni}_{51} \mathrm{Mn}_{24} \mathrm{Ga}_{25}$ a) case & 2.6 & 2.6 & 10.7 & 10.7 \\
\hline \multirow[t]{2}{*}{2} & $\mathrm{Ni}_{49.4} \mathrm{Mn}_{27.7} \mathrm{Ga}_{22.9} \mathrm{~b}$ ) case & 3.6 & 4.6 & 13.14 & 16.6 \\
\hline & $\mathrm{Ni}_{49.4} \mathrm{Mn}_{27.7} \mathrm{Ga}_{22.9}$ a) case & 3.6 & 4.8 & 13.14 & 17.7 \\
\hline 3 & $\mathrm{Ni}_{51.5} \mathrm{Mn}_{24.9} \mathrm{Ga}_{23.6} \mathrm{~b}$ ) case & 3.8 & 4.2 & 13.1 & 14.6 \\
\hline 4 & $\left.\mathrm{Ni}_{52.6} \mathrm{Mn}_{23.6} \mathrm{Ga}_{23.8} \mathrm{~b}\right)$ case & 4.0 & 5.3 & 14.3 & 18.8 \\
\hline 5 & $\left.\mathrm{Ni}_{52.6} \mathrm{Mn}_{23.9} \mathrm{Ga}_{23.5} \mathrm{~b}\right)$ case & 4.2 & 4.9 & 14.4 & 16.8 \\
\hline \multirow[t]{2}{*}{6} & $\left.\mathrm{Ni}_{52.0} \mathrm{Mn}_{24.4} \mathrm{Ga}_{23.6} \mathrm{~b}\right)$ case & 5.0 & 5.1 & 16.1 & 16.3 \\
\hline & $\mathrm{Ni}_{52.0} \mathrm{Mn}_{24.4} \mathrm{Ga}_{23.6}$ a) case & 5.0 & 8.1 & 16.1 & 26.3 \\
\hline 7 & $\mathrm{Ni}_{57.5} \mathrm{Mn}_{22.5} \mathrm{Ga}_{20}$ & 10 & 15.9 & 16.5 & 26.2 \\
\hline 8 & $\mathrm{Ni}_{53.1} \mathrm{Mn}_{26.6} \mathrm{Ga}_{20.3}$ & 11 & 11.38 & 29.3 & 31.5 \\
\hline 9 & $\mathrm{Ni}_{51.2} \mathrm{Mn}_{31.1} \mathrm{Ga}_{17.7}$ & 13 & 20.3 & 30.2 & 47 \\
\hline
\end{tabular}

Let us focus our attention on alloy 2 from Table 1, for which the experimental data under compression along [100] (case a), and [110] (case b) crystallographic axis are available. For this alloy, $Q<Q_{\text {calc }}{ }^{\mathrm{b}}<Q_{\text {calc }}{ }^{\mathrm{a}}$; thus, the Helmholtz free energies (assuming the same value for the free energy of austenite) are ordered in the sequence $\left.F>F^{\mathrm{b}}\right)>F^{\mathrm{a}}$. Here, $F$ is the energy of the three-variant state arising on cooling of the alloy, $\left.F^{b}\right)$ and $F^{a)}$ are the energies of the two- and single-variant states induced by the compressive force applied in [110] and [100] directions, respectively. It can therefore be concluded that the presence of an increasing number of variants and corresponding lattice misfit prevents states of lower energy from being achieved.

It is worth noting that, in the case of transformation to a lattice with $c / a>1$ (alloys 7-9), the values of $Q_{\text {calc }}$ are larger than for alloys transforming to a martensite with $c / a<1$. This is properly explained by Equation (4), taking into account the experimental values $|1-c / a|<0.1$ reported for the alloys $1-6$, with $c / a<1$ and $|1-c / a| \approx 0.2$ obtained for the alloys 7-9 with $c / a>1$ (see [12] and references 
therein). Thus, it can be clearly seen that the large values of $Q$ and $\Delta S$ measured for the alloys with $c / a>1$ are related to large values of MT strain. It can be deduced that for the Ni-Mn-Ga martensites with $c / a>1$, the elastic part of $\Delta S$ exceeds its magnetic part, while for those with $c / a<1$, the ratio of elastic and magnetic parts may be grater or smaller than unity, depending on the value $T_{C A}-T_{M}$.

As was argued above, the separation of the magnetic parts from the total entropy change and transformation heat requires quantitative theoretical calculations. The theoretical expression for $\Delta S_{m a g}$ (see Equation (2)) was obtained in Reference [7] from the minimum conditions for the magnetoelastic part of the free energy, which depends on the magnetization of the alloy, the transformation strains, and the volume change during MT. The magnetic contribution to the transformation heat can be expressed as a function of $T_{C A}-T_{M F}$ using the relationship $Q_{m a g}=T_{M} \Delta S_{m a g}$. As a study case, the $\Delta S_{\text {mag }}$ value was computed for the $\mathrm{Ni}_{52.6} \mathrm{Mn}_{23.5} \mathrm{Ga}_{23.9}$ at \% alloy (Alloy A). To avoid computational difficulties arising in the course of differentiation of a discrete experimental function $M_{\exp }(T)$, this function was approximated by the solutions of equations

$$
M(T)= \begin{cases}\tanh \left[\frac{T_{C A} M(T)}{T M(0)}\right], & \text { if } T>T_{M S} \\ \tanh \left[\frac{T^{*} M(T)}{T M(0)}\right], & \text { if } T<T_{M F}\end{cases}
$$

where $T^{*}=T_{C A}\left[1+6\left(\delta_{e x} / \zeta\right) v_{M T}\right]$, and $\zeta / T_{C A}$ is the temperature derivative of the magnetic exchange parameter. The dimensionless parameter $\kappa=2 \delta_{e x} v_{M T} / 3$ can be introduced in Equation (2), yielding a function that depends on this parameter, the function $M(T)$, and its derivative. This parameter is present in Equation (5) for magnetization, because it is easy to see that $T^{*}=(1+9 \kappa / \zeta) T_{C A}$. The values $M(0) \approx 715 \mathrm{G}$ and $\zeta M^{2}(0) \approx 0.1 \mathrm{GPa}$ have been reported for alloy [20]. The $M(T)$ function was computed and fitted to experimental magnetization values. The value $\kappa$ was considered a fitting parameter [7]. Equation (5) provided an excellent fit of the theoretical $M(T)$ curve to the experimental points in both austenitic and martensitic state for $\kappa=150$ (see Figure 1).

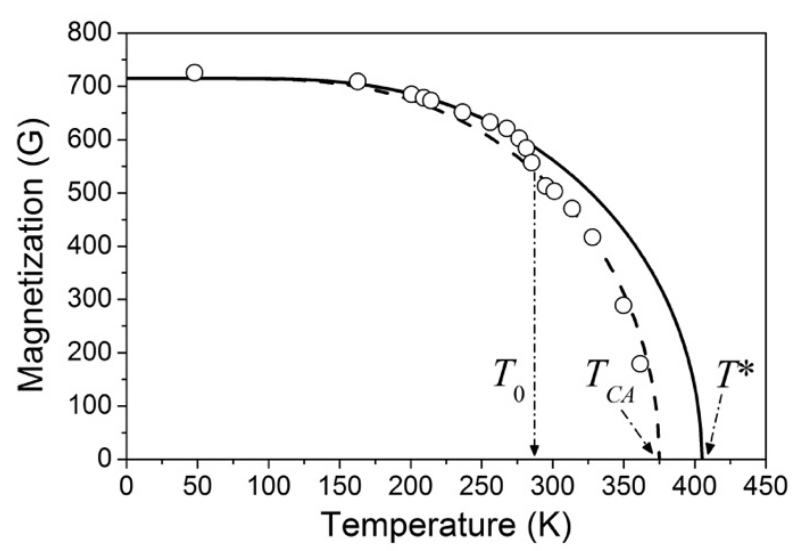

Figure 1. Saturation magnetization of Alloy A computed from Equation (5) (lines) and measured in Reference [21] (open circles). The dashed and solid lines are fitted to the magnetization values measured for the austenitic and martensitic phases, respectively. The fitting was performed by the proper choice of $T_{C A}$ value and parameter $\kappa$ in the formula for $T^{*}$. The dependence shown by the solid line is used for computation of the magnetic entropy change.

This value of parameter $\kappa$ results in a $\Delta S_{m a g}=14.3 \mathrm{~J} / \mathrm{kg} \mathrm{K}$, which corresponds to a $Q_{\text {mag }}=4.1 \mathrm{~J} / \mathrm{g}$, with $T_{M} \approx 285 \mathrm{~K}$ for this alloy. The estimated $Q_{m a g}$ is similar to the experimental value of the transformation heat $Q \approx 4.2 \mathrm{~J} / \mathrm{g}$ reported for this alloy [22,23]. It can be concluded, therefore, that the magnetic subsystem of this alloy is the main contributor to transformation heat and entropy change.

Substituting the material constants estimated for Alloy A into Equations (2) and (5), and keeping values of $T_{M S}-T_{M F}$ and $T_{C A}$ constant, one can obtain the plots of the functions $\Delta S_{m a g}\left(T_{C A}-T_{M}\right)$ and 
$Q_{m a g}\left(T_{C A}-T_{M}\right)$ by changing the value of $T_{M}=\left(T_{M S}+T_{M F}\right) / 2$. These functions are shown by the solid lines in Figure 2a,b, respectively, in comparison with experimental values of the transformation heat $Q$ measured for several Ni-Mn-Ga alloys with $c / a<1$ [15,24-27]. There is a good correlation between the experimental values of $Q$ and the computed $Q_{m a g}$, in spite of the fact that the dependences $M(T)$ are different for different Ni-Mn-Ga alloys, whereas the calculated dependences are based on the experimental data obtained for Alloy A (Figure 1).

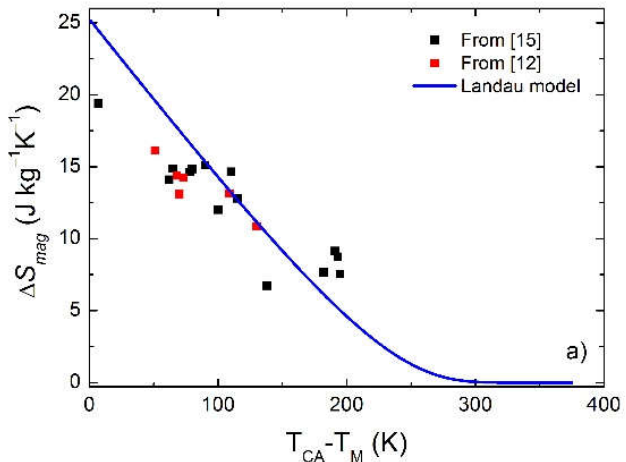

(a)

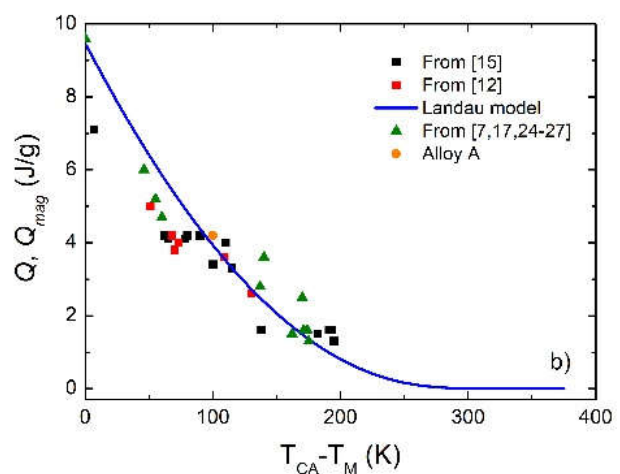

(b)

Figure 2. (a) Calculated magnetic entropy change versus $T_{C A}-T_{M}$ (solid line) and complete entropy changes (symbols) measured for different Ni-Mn-Ga alloys; (b) Calculated magnetic contribution to the transformation heat versus $T_{C}-T_{M}$ (solid line) and transformation heats (symbols) measured for different Ni-Mn-Ga alloys.

Figure 2 shows the calculated magnetic contributions to entropy change and heat exchange in the course of MT, compared with the total experimental values obtained for different Ni-Mn-Ga alloys. The calculated magnetic contributions are close to the experimental values, or even exceed them when $T_{C A}-T_{M}<120 \mathrm{~K}$. If the opposite inequality is fulfilled, the majority of the total experimental values noticeably exceed the calculated magnetic contribution. In any case, from Figure 2, it seems clear that the magnetic contributions to $\Delta S$ and $Q$ are the most important for Ni-Mn-Ga alloys with $T_{M F}<T_{C A}$, and that these contributions increase sharply when $T_{C A}-T_{M} \rightarrow 0$. This feature of transformational behavior of Ni-Mn-Ga is similar to that observed in metamagnetic alloys, such as Ni-Mn-In or Ni-Mn-In-Co, where $\Delta S$ also increases with decreasing $T_{C}-T_{M}[9,28]$. However, it has to be kept in mind that in metamagnetic alloys, the MT takes place usually from ferromagnetic austenite to para- or weakly-magnetic martensite, and it is to be expected that $\Delta S_{\text {mag }}=S_{A \text { mag }}-S_{M \text { mag }}<0$. At the same time, as the direct transformation is exothermic, the inequality $S_{M}<S_{A}$ takes place, and in accordance with the notation accepted above, $\Delta S=\left|S_{M}-S_{A}\right|=S_{A}-S_{M}>0$. It has been shown that the increase of $\Delta S$ is mostly due to the decreasing magnetic order of austenite as $T_{M}$ approaches $T_{C A}[10]$.

The $Q_{\text {mag }}$ values estimated from the function $Q_{m a g}\left(T_{C A}-T_{M F}\right)$ for the alloys listed in Table 1 are presented in Table 2. (The characteristic temperatures $T_{C}$ and $T_{M}$ measured for these alloys are collected in the Appendix to Reference [12]; the function $Q_{m a g}\left(T_{C A}-T_{M F}\right)$ was computed in [7].) 
Table 2. Magnetic contribution to the transformation heat $\left(Q_{m a g}\right)$, and heat differences between the total transformation heat and magnetic contribution calculated for both experimental $(Q)$ and calculated $\left(Q_{\text {calc }}\right)$ total heats.

\begin{tabular}{cccc}
\hline Alloy & $Q_{\text {mag }}(\mathrm{J} / \mathrm{g})$ & $Q-Q_{\text {mag }}(\mathrm{J} / \mathrm{g})$ & $Q_{\text {calc }}-Q_{\text {mag }}(\mathrm{J} / \mathrm{g})$ \\
\hline 1 & 2.5 & 0.1 & 0.1 \\
2 a) case & 3.5 & 0.1 & 1.1 \\
b) case & 3.5 & 0.1 & 1.3 \\
3 & 5.3 & -1.5 & -1.1 \\
4 & 5.1 & -1.1 & 0.2 \\
5 & 5.4 & -1.2 & -0.5 \\
6 a) case & 6.3 & -1.3 & -1.2 \\
b) case & 6.3 & -1.3 & 1.8 \\
\hline
\end{tabular}

Considering the data presented in Table 2, it is worth remembering that the expression for $\Delta S_{\text {mag }}$, which was used for evaluation of $Q_{m a g}$, corresponds to the minimum of the magnetoelastic part of the energy, while minimum elastic energy (and, therefore, maximum experimentally measured transformation heat $Q$ ) is not reached in the course of real MTs. As such, negative values appear for $Q-Q_{m a g}$ and $Q_{\text {calc }}-Q_{m a g}$. Comparing the values of $Q_{m a g}$ with the differences $Q-Q_{m a g}$ and $Q_{\text {calc }}-Q_{\text {mag }}$, which correspond to the lattice contribution of heat exchange, it seems obvious that the majority of the entropy change during the MT is related to the magnetic order when the MT takes place between ferromagnetic phases. Nevertheless, one has to keep in mind that the consideration of transformation heat as the sum of magnetic and elastic parts is a formal procedure (and has been shown to be useful to estimate the weight of these components). It should be concluded that, physically, the elastic properties of FSMAs are strongly influenced by the magnetic order, and vice versa, the appearance of MT strain upon the cooling of FSMA strongly changes its magnetic characteristics (magnetic exchange energy, magnetic anisotropy constants, ferromagnetic resonance frequencies, and so on).

The general conclusion presented above is supported by the interesting result obtained within the frame of the Landau theory of phase transitions in FSMA as being used for the calculation of $\Delta S$ and $\Delta S_{m a g}$ [7]. Disregarding the anisotropic part of the ordinary magnetostriction in the spontaneous strain $\varepsilon_{M}$ arising in the course of cubic-tetragonal MT and the magnetoelastic part of the free energy, one can obtain (see Reference [7]), the relationship

$$
T_{M S}-T_{M F} \approx \frac{1}{\Delta S} \cdot \frac{3 \varepsilon_{M}^{2} C^{\prime}\left(T_{M S}\right)}{2 m},
$$

where $m$ is the mass density. This relationship shows that the width of the temperature interval of MT is inversely proportional to $\Delta S$. Equation (6) has been derived for cases where there is no axial mechanical stress. Its solution, obtained using $\varepsilon_{M}=0.04, m=8 \mathrm{~g} \cdot \mathrm{cm}^{-3}$ and $C^{\prime}\left(T_{M S}\right)=1 \mathrm{GPa}$, is presented in Figure 3 by the solid line. For Ni-Mn-Ga alloys, there are enough theoretical and experimental data to allow the application of Equation (6). As an example, for Alloy A, $\Delta S=14.7 \mathrm{~J} / \mathrm{kg} \cdot \mathrm{K}$ and the experimental values given for $T_{M S}$ and $T_{M F}$ give $T_{M S}-T_{M F} \approx 8 \mathrm{~K}$, whereas Equation (6) results in the estimation $T_{M S}-T_{M F} \approx 21 \mathrm{~K}$ (see upper arrow in Figure 3). Therefore, it should be taken into account that the martensitic transformation occurs in the ferromagnetic phase of alloy, and so the magnetoelastic term, which describes the axial magnetostriction, must be included in the Landau expansion for Helmholtz free energy. This term involves the magnetoelastic stress, which causes axial magnetostriction. Appropriate computations showed that magnetoelastic stress reduces the temperature interval of MT in Alloy A to experimental value (see dashed line and arrow) [7]. 


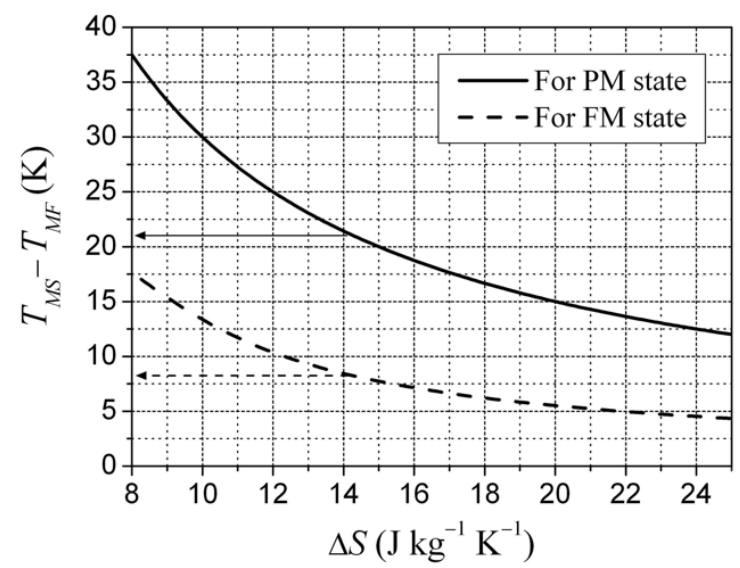

Figure 3. Temperature interval of MT as a function of the entropy change computed for the paramagnetic and ferromagnetic state of shape memory alloy (i.e., for zero and non-zero values of magnetoelastic stress).

\section{Concluding Remarks}

Some important remarks, which may inspire new experiments, are as follows:

(a) Experiments carried out for different alloy compositions confirm the linear dependence of $T_{M S}-T_{M F}$ on $\Delta S^{-1}$. This shows that the product $\varepsilon_{M}^{2} C^{\prime}\left(T_{M S}\right)$ weakly depends on the composition of the alloy.

(b) It is worth noting that a shear modulus value of 1 GPa gives the correct theoretical estimation for $T_{M S}-T_{M F}$. The value $C^{\prime}=1 \mathrm{GPa}$ is a lower value than that resulting from numerous experiments; given that the choices for $\varepsilon_{M}=0.04$ and $m=8 \mathrm{~g} \cdot \mathrm{cm}^{-3}$ are properly defined, choosing a higher value for $C^{\prime}$ would lead to non-realistic $T_{M S}-T_{M F}$ differences. However, a pronounced tendency takes place: the experimental values of the elastic moduli of different FSMAs reported in the recent works are lower than the values measured previously. For example, the values of elastic modulus $\sim 2$ GPa were registered in $\mathrm{Ni}_{29} \mathrm{Mn}_{48} \mathrm{Ga}_{23}$ single crystal using dynamic mechanical analysis [29]; a linear decrease of shear modulus on approaching the temperature of $\mathrm{Fe}_{3} \mathrm{Pt}$ alloy was reported very recently, the extrapolation of the experimental line to $T_{M S}$ resulted in the estimation $C^{\prime}\left(T_{M S}\right) \sim 0.6 \mathrm{GPa}$ [30].

(c) A strong increase of $\Delta S^{-1}$ with $\left(T_{M S}-T_{M F}\right)$ was observed in metamagnetic alloys such as Ni-Mn-In and Ni-Mn-(In,Sn)-Co, see Figure 4. Nevertheless, the inverse relationship, as predicted by Equation (6), was found to be fulfilled only for transformations under magnetic field.

(d) The entropy changes caused by the MTs of different FSMAs were previously estimated from the results of experimental stress-strain tests performed at different temperatures, or from the measurements of elastic moduli (that is, from the experimental values $d \sigma_{M S} / d T$ and $d C^{\prime} / d T$, respectively). For the correct interpretation of experimental results, it should be remembered that, in cases where MT occurs in the ferromagnetic phase of alloy, the estimated entropy change is the sum of $\Delta S_{e l}$ and $\Delta S_{\text {mag. }}$.

(e) A thermodynamic analysis of existing experimental data was carried out above, without taking into account the five-layered and seven-layered modulation of crystal lattice inherent to Ni-Mn-Ga martensites. The equations, which include the temperature derivative of the stress value initiating the MT, were used. As far as we know, the theoretical analysis of the influence of lattice modulation on this derivative is an open problem. 


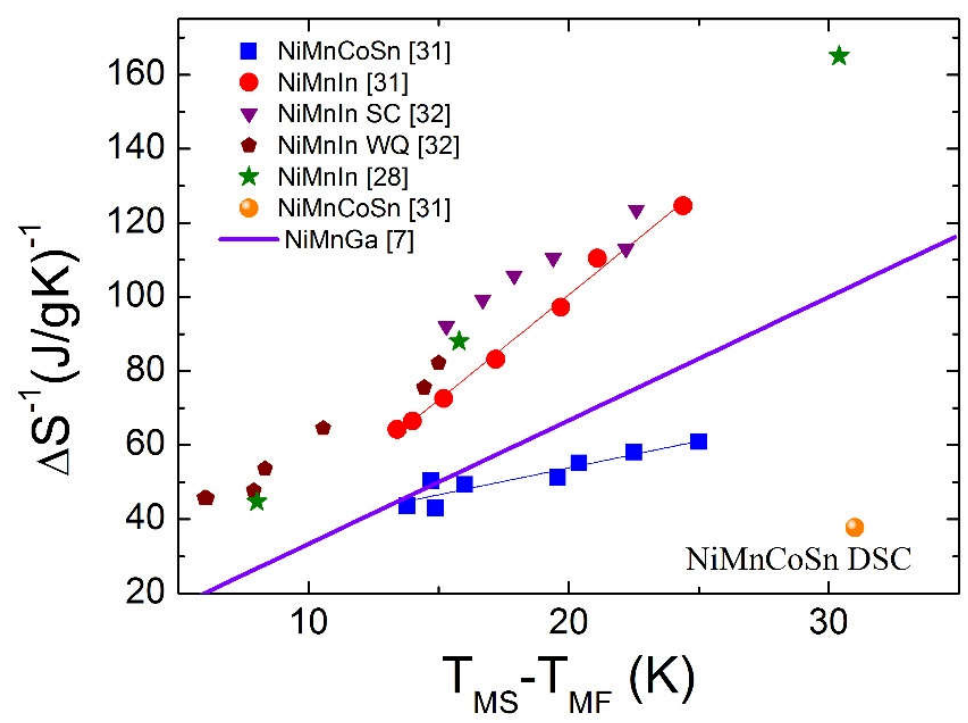

Figure 4. The reciprocal values of entropy change during MT measured for metamagnetic alloys (symbols). Theoretical values obtained for Ni-Mn-Ga alloy system are presented for comparison by the solid line. Thin lines are guides for the eye [7,28,31,32].

Acknowledgments: Partial financial support from projects MAT2014-56116-C04-01-R and MAT2012-37923-C02-01 $(A E I / F E D E R, U E)$ is acknowledged. The financial support from project 0117U000433 of NASU and project 0117U004340 of MESU is also acknowledged.

Author Contributions: Joan Torrens-Serra, Vicente Recarte and José Ignacio Pérez-Landazábal performed the experiments and arranged the experimental data. Victor A. L'vov, Eduard Cesari and Anna Kosogor analyzed the experimental data and wrote the paper.

Conflicts of Interest: The authors declare no conflict of interest.

\section{References}

1. Ullakko, K.; Huang, J.K.; Kantner, C.; O’Handley, R.C.; Kokorin, V.V. Large magnetic-field-induced strains in $\mathrm{Ni}_{2} \mathrm{MnGa}$ single crystals. Appl. Phys. Lett. 1996, 69, 1966-1968. [CrossRef]

2. O'Handley, R.C.; Murray, S.J.; Marioni, M.; Nembach, H.; Allen, S.M. Phenomenology of giant magnetic-field-induced strain in ferromagnetic shape-memory materials (invited). J. Appl. Phys. 2000, 87, 4712-4717. [CrossRef]

3. Sozinov, A.; Likhachev, A.A.; Lanska, N.; Ullakko, K. Giant magnetic-field-induced strain in NiMnGa seven-layered martensitic phase. Appl. Phys. Lett. 2002, 80, 1746-1748. [CrossRef]

4. Kainuma, R.; Imano, Y.; Ito, W.; Sutou, Y.; Morito, H.; Okamoto, S.; Kitakami, O.; Oikawa, K.; Fujita, A.; Kanomata, T.; et al. Magnetic-field-induced shape recovery by reverse phase transformation. Nature 2006, 439, 957-960. [CrossRef] [PubMed]

5. Chatterjee, S.; Giri, S.; Majundar, S.; De, S.K. Giant magnetoresistance and large inverse magnetocaloric effect in $\mathrm{Ni}_{2} \mathrm{Mn}_{1.36} \mathrm{Sn}_{0.64}$ alloy. J. Phys. D Appl. Phys. 2009, 42. [CrossRef]

6. Karaca, E.; Karaman, I.; Basaran, B.; Ren, Y.; Chumlyakov, Y.I.; Maier, H.J. Magnetic field-induced phase transformation in NiMnCoIn magnetic shape-memory alloys-A new actuation mechanism with large work output. Adv. Funct. Mater. 2009, 19, 983-998. [CrossRef]

7. L'vov, V.A.; Cesari, E.; Recarte, V.; Perez-Landazabal, J.I. Entropy change of martensitic transformation in ferromagnetic shape memory alloys. Acta Mater. 2013, 61, 1764-1772. [CrossRef]

8. Ito, W.; Imano, Y.; Kainuma, R.; Sutou, Y.; Oikawa, K.; Ishida, K. Martensitic and magnetic transformation behaviors in heusler-type NiMnIn and NiCoMnIn metamagnetic shape memory alloys. Metall. Mater. Trans. A 2007, 38, 759-766. [CrossRef] 
9. Kustov, S.; Corró, M.L.; Pons, J.; Cesari, E. Entropy change and effect of magnetic field on martensitic transformation in a metamagnetic Ni-Co-Mn-In shape memory alloy. Appl. Phys. Lett. 2009, 94. [CrossRef]

10. Pérez-Sierra, A.M.; Bruno, N.M.; Pons, J.; Cesari, E.; Karaman, I. Atomic order and martensitic transformation entropy change in Ni-Co-Mn-In metamagnetic shape memory alloys. Scr. Mater. 2016, 110, 61-64. [CrossRef]

11. Chernenko, V.A.; L'vov, V.A.; Cesari, E.; Kosogor, A.; Barandiaran, J.M. Transformation volume effects on shape memory alloys. Metals 2013, 3, 237-282. [CrossRef]

12. Kosogor, A.; L'vov, V.A.; Cesari, E. Influence of volume magnetostriction on the thermodynamic properties of Ni-Mn-Ga shape memory alloys. J. Appl. Phys. 2015, 118. [CrossRef]

13. Chernenko, V.A.; Pons, J.; Seguí, C.; Cesari, E. Premartensitic phenomena and other phase transformations in Ni-Mn-Ga alloys studied by dynamical mechanical analysis and electron diffraction. Acta Mater. 2002, 50, 53-60. [CrossRef]

14. Danilevich, A.G.; L'vov, V.A. Strong influence of ferromagnetic ordering and internal pressure on the elastic modulus of shape memory alloy. J. Magn. Magn. Mater. 2013, 333, 108-113. [CrossRef]

15. Chernenko, V.A.; Cesari, E.; Kokorin, V.V.; Vitenko, I.N. The development new ferromagnetic shape memory alloys in Ni-Mn-Ga system. Scr. Metall. Mater. 1995, 33, 1239-1244. [CrossRef]

16. Fabricci, S.; Karamad, J.; Arnold, Z.; Casoli, F.; Palouzi, A.; Bolzoni, F.; Cabassi, R.; Solzi, M.; Porcari, G.; Pernechele, C.; Albertini, F. From direct to inverse giant magnetocaloric effect in Co-doped NiMnGa multifunctional alloys. Acta Mater. 2011, 59, 412-419. [CrossRef]

17. Chernenko, V.A.; Cesari, E.; Pons, J.; Segui, C. Phase transformations in rapidly quenched Ni-Mn-Ga alloys. J. Mater. Res. 2000, 15, 1496-1504. [CrossRef]

18. Webster, P.J.; Ziebeck, K.R.A.; Town, S.L.; Peak, M.S. Magnetic order and phase transformation in $\mathrm{Ni}_{2} \mathrm{MnGa}$. Philos. Mag. B 1984, 49, 295-310. [CrossRef]

19. Chernenko, V.A.; L'vov, V.A.; Zagorodnyuk, S.P.; Takagi, T. Ferromagnetism of thermoelastic martensites: Theory and experiment. Phys. Rev. B 2003, 67, 064407. [CrossRef]

20. Chernenko, V.A.; L'vov, V.A. Magnetoelastic nature of ferromagnetic shape memory effect. Mater. Sci. Forum 2008, 583, 1-20. [CrossRef]

21. Kokorin, V.V.; Chemenko, V.A.; Val'kov, V.I.; Konoplyuk, S.M.; Khapaliuk, E.A. Magnetic transitions in $\mathrm{Ni}_{2} \mathrm{MnGa}$. Phys. Solid State 1995, 37, 2049-2051.

22. Chernenko, V.A.; Kokorin, V.V.; Babiy, O.M.; Zasimchuk, I.K. Phase diagrams in the Ni-Mn-Ga system under compression. Intermetallics 1998, 6, 29-34. [CrossRef]

23. Chernenko, V.A.; Amengual, A.; Cesari, E.; Kokorin, V.V.; Zasimchuk, I.K. Thermal and magnetic properties of stress-induced martensites in Ni-Mn-Ga alloys. J. Phys. IV Fr. 1995, 5, C2-95-C2-98. [CrossRef]

24. Fukuda, T.; Maeda, H.; Yasui, M.; Kakeshita, T. Influence of magnetocrystalline anisotropy on martensitic transformation under magnetic field of single-crystalline $\mathrm{Ni}_{2} \mathrm{MnGa}$. Scr. Mater. 2009, 60, 261-263. [CrossRef]

25. Seguí, C.; Chernenko, V.A.; Pons, J.; Cesari, E. Low-temperature-induced intermartensitic phase transformations in Ni-Mn-Ga single crystal. J. Magn. Magn. Mater. 2005, 290, 811-815. [CrossRef]

26. Seguí, C.; Chernenko, V.A.; Pons, J.; Cesari, E. Two-step martensitic transformation in Ni-Mn-Ga alloys. J. Phys. IV Fr. 2003, 112, 903-906. [CrossRef]

27. Khovailo, V.V.; Takagi, T.; Tani, J.; Levitin, R.Z.; Cherechukin, A.A.; Matsumoto, M.; Note, R. Magnetic properties of $\mathrm{Ni}_{2.18} \mathrm{Mn}_{0.82} \mathrm{Ga}$ Heusler alloys with a coupled magnetostructural transition. Phys. Rev. B 2002, 65. [CrossRef]

28. Recarte, V.; Pérez-Landazábal, J.I.; Sánchez-Alarcos, V.; Zablotskii, V.; Cesari, E.; Kustov, S. Entropy change linked to the martensitic transformation in metamagnetic shape memory alloys. Acta Mater. 2012, 60, 3168-3175. [CrossRef]

29. Chernenko, V.A.; Villa, E.; Salazar, D.; Barandiaran, J.M. Large tensile superelasticity from intermartensitic transformations in $\mathrm{Ni}_{49} \mathrm{Mn}_{28} \mathrm{Ga}_{23}$ single crystal. Appl. Phys. Lett. 2016, 108. [CrossRef]

30. Fukuda, T.; Kakeshita, T. Lattice softening in $\mathrm{Fe}_{3} \mathrm{Pt}$ exhibiting three types of martensitic transformations. Metals 2017, 7, 156. [CrossRef] 
31. Lázpita, P.; Sasmaz, M.; Cesari, E.; Barandiarán, J.M.; Gutierrez, J.; Chernenko, V.A. Martensitic transformation and magnetic field induced effects in $\mathrm{Ni}_{42} \mathrm{Co}_{8} \mathrm{Mn}_{39} \mathrm{Sn}_{11}$ metamagnetic shape memory alloy. Acta Mater. 2016, 109, 170-176. [CrossRef]

32. Barandiarán, J.M.; Chernenko, V.A.; Cesari, E.; Salas, D.; Gutierrez, J.; Lázpita, P. Magnetic field and atomic order effect on the martensitic transformation of a metamagnetic alloy. J. Phys. Condens. Matter 2013, 25, 484005. [CrossRef] [PubMed]

2017 by the authors. Licensee MDPI, Basel, Switzerland. This article is an open access article distributed under the terms and conditions of the Creative Commons Attribution (CC BY) license (http://creativecommons.org/licenses/by/4.0/). 